\title{
Clinical association between teeth malocclusions, wrong posture and ocular convergence disorders: an epidemiological investigation on primary school children
}

Armando Silvestrini-Biavati ${ }^{1}$, Marco Migliorati ${ }^{1}$, Eleonora Demarziani ${ }^{2}$, Simona Tecco ${ }^{3}$, Piero Silvestrini-Biavati ${ }^{4}$, Antonella Polimeni $i^{5}$ and Matteo Saccucci ${ }^{5^{*}}$

\begin{abstract}
Background: As the various systems in the body are inter-connected to form a single structural unit, a pathological condition in one area can also affect other areas. There are many known correlations between the visual and motor system. The importance of visual function, particularly the paracentral peripheral field of view, in motor coordination, ambulation and the maintenance of balance has been amply demonstrated.

In line with current medical principles, which are moving towards a more holistic view of the human body, this study aims to investigate, in an interdisciplinary manner, the incidence of dental malocclusions together with posture and eye convergence disorders.

Methods: Six hundred and five children attending at the $3^{\text {rd }}, 4^{\text {th }}$ and $5^{\text {th }}$ years of seven Genoa primary schools were examined. Each child underwent the following examinations: (i) dental/occlusal; (ii) orthoptic; and (iii) postural. Occlusal data concerned the presence of cross-bite, midline deviation with a mandibular shift, bad habits and deep or open bite.

Postural assessment involved frontal and lateral inspection, investigation during trunk flexion and ambulation, and note of any asymmetry in the lower limbs. The recorded orthoptic data included those pertaining to ocular dominance, a cover test, convergence and the Brock string test.

Results: A prevalence of cases with an unphysiological gait was found in patients with overjet (14.70\%) or overbite (14.87\%), while the percentage of patients with normal occlusion that showed an unphysiological gait was 13.08\%. Also, about $93.8 \%-94.2 \%$ of children showed normal legs without dysmetry, with no difference in respect to the type of occlusion. Subjects with an open bite or deep bite showed a slightly different distribution of right or left dominant eyes.

Conclusion: About 13\% of children showed a pathological gait and, among them, vertical anomalies of occlusion (deep bite or open bite) were prevalent with respect to the other occlusal defects. The vertical dimension of occlusion revealed a slight relationship with the proper dominant eye. Postural, orthoptic, osteopathic and occlusal variables were often clinically associated, and therefore these disorders appear to request a multidisciplinary medical approach for their treatment.
\end{abstract}

Keywords: Teeth malocclusions, Wrong posture, Ocular convergence disorders

\footnotetext{
* Correspondence: m.sacca@hotmail.it

${ }^{5}$ Department of Pedodontics, Sapienza University of Rome, Rome, Italy

Full list of author information is available at the end of the article
} 


\section{Background}

As the various systems in the body are inter-connected to form a single structural unit, a pathological condition in one area can also affect other areas. In particular, the skeletal muscles play a decisive role in the coincidence of various disorders, because of the continuous anatomical and functional "chain" they form between the skull, lower jaw, spine, limbs and pelvis [1-5].

Indeed, if a situation of muscular high tension arises in one of the links in this chain (mandible, hyoid, vertebrae, pelvis and limbs), it is immediately transmitted to the rest of the body. As a consequence, the body loses its state of equilibrium, giving rise to compensation mechanisms, for example muscular tension in other antagonistic parts of the body. In this way, dental malocclusion can be associated with misalignment of the mandible, one of the links in the muscular chain, leading to hyper-contraction of the masticatory muscles [6]. This tension forces the rest of the body to react, imposing postural modifications brought about by the contraction of other muscles in the chain. In fact, the incidence of malocclusion in orthopedic patients reported in the literature ranges from $83 \%$ to $87 \%$ [7]. Furthermore, it is now widely recognized that problems involving the stomatognathic apparatus and alterations in feet posture can cause kinetic dysfunction, leading to pathologies in the ascending and descending spinal tracts $[7,8]$.

Perinetti et al. [9] investigated malocclusal traits correlated with body posture alterations. A limited number of significant correlations were observed, mainly for overbite, when using multivariate models.

Moreover, it has become increasingly evident that disorders of the sensory nervous system can have a considerable influence on regulation of motor function [8]. In fact, modifications in these sensory neurons can cause parafunctional alterations and pathologies in apparently unrelated body districts, For instance, dental malocclusion can cause pain elsewhere in the body. Because of its position, misalignment of the mandible can also cause the position of the pupillary line to be momentarily altered, provoking the intervention of ocular muscles to keep the gaze straight.

There are many known correlations between the visual and motor systems, and the importance of visual function, particularly the paracentral peripheral field of view, in motor coordination, ambulation and the maintenance of balance has been amply demonstrated [10]. In fact, to follow an object in motion, the eye needs to be able to coordinate the movement of the head and neck. The musculature controlling eye movement is closely connected to the stomatognathic system. Fibers emerging from the muscle spindles and palisade endings in the oculomotor muscles, especially the lateral rectus muscle, form pathways to the oculomotor nuclei and the trigeminal nucleus [11-13]. Ocular defects that can be linked to dental malocclusion are convergence defects, heterophoria, heterotrophia and esodeviations.

This study is based on three presuppositions: 1) a mandibular shift may cause a positional head adaptation [13]; 2) ocular phorias may cause a head compensation posture called ocular torticollis [14]; and 3) a head compensation posture causes body adaptation positions, to maintain a center of gravity compatible with the upright position [15]. Head positions enhance postural compensation phenomena; thereby head position is a key point in body balance. This is demonstrated by the great incidence of cervical pain due to muscular tension, which means head posture controls hyperfunctions $[16,17]$.

In this study, the aim was to determine if these disorders occur with significant frequency in children (aged 7-10 years). We analyzed in primis dental malocclusions (by an orthodontist) and subsequently orthophoria was recorded (by an orthoptist). Then the cranio-sacral respiratory rhythm was tested (by an osteopath). The incidence and concomitant frequency of these disorders were statistically analyzed to understand whether a causal connection existed in situations where patients were forced to carry out functional compensations in different body districts.

\section{Methods}

In this epidemiological study, conducted with the collaboration of the local health authority (ASL $3,1^{\text {st }}$ District, School Medicine Services), 605 children (mean age $8.5 \pm$ 2.3 years; $45 \%$ males; $55 \%$ females) attending the $3^{\text {rd }}, 4^{\text {th }}$ and $5^{\text {th }}$ years of seven Genoa primary schools were examined.

Before any clinical research was performed, the parents of the children attending the selected classes were asked to provide informed consent and to fill in an anamnestic survey (i.e., birth, breast and/or formula feeding habits, dummy- or thumb-sucking, headaches, dental and general trauma, pain in the muscles of the head and neck and nocturnal bruxism). The questionnaire pertaining to each child was then used at subsequent clinical examinations to notify the medical specialists of any related disorders, thereby assisting them in the formation of an accurate clinical picture. During this screening, each child underwent the following examinations: 1) dental/occlusal; 2) orthoptic; and 3) osteopathic-postural. These investigations were performed at the school by two dentists, one orthoptist and one osteopathist, under the expert supervision of a clinician specialized in School Medicine (from the ASL $3,1^{\text {st }}$ District). The examination protocol adopted was based on previous studies [16,17]. 


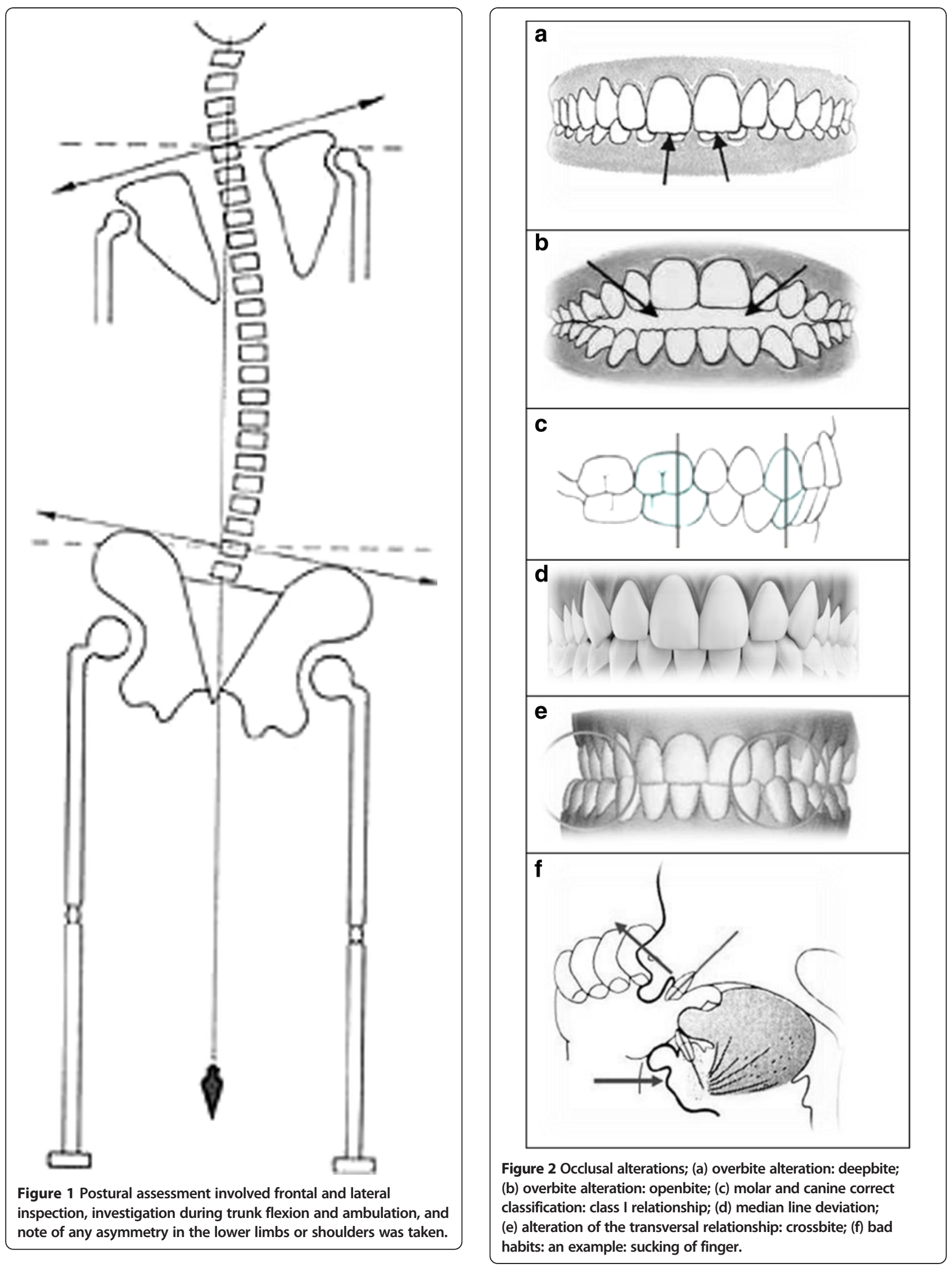




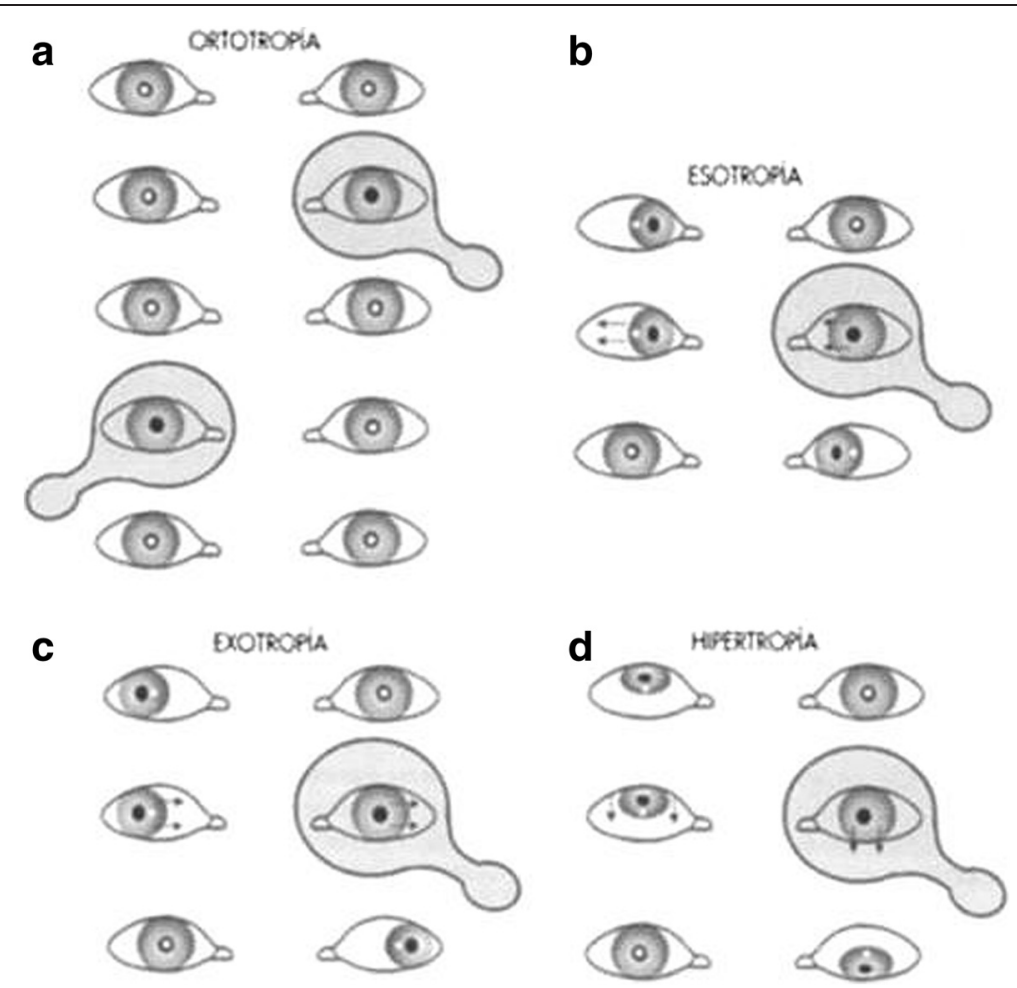

Figure 3 Cover test for eyes; (a) ortotrophia; (b) esotrophia; (c) exotrophia; (d) hipertrophia.

Three clinical evaluation forms that were previously designed were used to record the dental, orthoptic and postural data, and a medical record of each child was compiled.

Postural assessment involved frontal and lateral inspection, investigation during trunk flexion and ambulation, and note of any asymmetry in the lower limbs was taken (Figure 1). The collected dental information contained details of the molar and canine relationships, dental and facial midline, overbite, transversal relationships (cross-bite presence/absence), bad habits, hygiene and ongoing orthodontic treatment (Figure 2). The recorded orthoptic data included those pertaining to a cover test, convergence, ocular dominance and the Brock string test (Figures 3, 4, 5).
The following eye tests were carried out primarily to determine postural information:

- Cover/uncover test (CT; Figure 3): The test was performed by covering and uncovering each eye in turn (e.g., with a hand or occluder) while the patient fixated on a small object. The purpose of the test was to determine any re-fixation movement in the uncovered eye. In this way, each eye was considered as a single entity and any axial defects (heterophoria) were detected by the presence of re-fixation movements in the uncovered eye (dissociation of the right and left eyes). An experienced ophthalmologist was then able to quantify the degree of phoria by passing a test card featuring

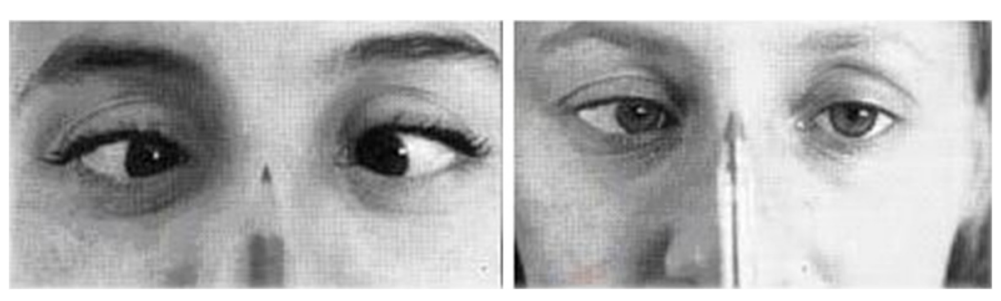

Figure 4 Convergence test. 


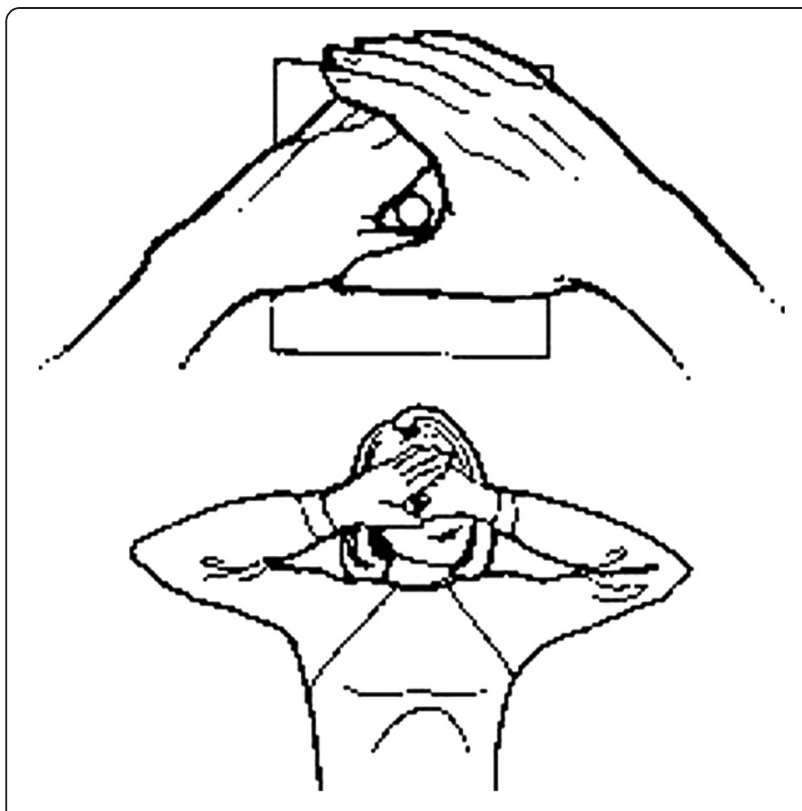

Figure 5 Dominant eye test.

increasing prism diopter values before the eyes until the re-fixation movement disappeared.

- Convergence test (CT; Figure 4): The test was performed up to the base of the nose to determine the degree of tonic (dynamic), fusional and accommodative convergence.

There are two types of convergence:

- Dynamic convergence (smooth pursuit system), which is considered pathological if the saccadic movements of one eye are slower than the other, the convergence movement of one or both eyes fails to complete, or the eye or eyes wander outwards when attempting to converge. Postural imbalance resulting from this condition will be greater if the defect is more pronounced in one eye.

- Reflexive convergence (saccadic system), which seems to involve the saccadic rather than pursuit system, and is considered pathological if an eye fails to converge, stops in transit or wanders outwards when attempting to perform convergence. If the two eyes contradict each other, the convergence defect is considered to be present in both eyes. If the hypoconvergent eye protrudes and points downwards during reflexive convergence, a phoria or trophia is likely to be present.

- Ocular dominance test (Figure 5): A card with a hole of diameter $2 \mathrm{~cm}$ is placed a distance of $10-15 \mathrm{~cm}$ from the patient's eyes. The patient is then asked to stare through the hole at a distant object and the eye that automatically complies is the dominant eye. Confirmation of ocular dominance can be achieved by covering each eye in turn and repeating the test. Without moving the pierced card, the dominant eye will be the only one able to see the object in the center of the hole.

Parents were present and fully informed about the clinical findings. All patients approved the inclusion of their data in this study. The research reported in the paper was undertaken in compliance with the Helsinki Declaration. Ethical approval was granted by the Ethics Committee of the S. Martino Hospital- University of Genoa-National Institute for Cancer Research (P.R 21/12). Written consent was obtained after orientation to the study.

\section{Statistical evaluation}

All data were analyzed in the form of percentages of subjects with a particular occlusal or postural or ocular situation. The percentages of subjects with postural or ocular diseases were compared among subjects with a normal, deep or open bite.

A chi-square test was used to evaluate the null hypothesis stating that the frequency distributions of gait posture, leg dysmetry or ocular diseases observed in our sample were consistent with a particular theoretical distribution. The null hypothesis was that the considered diseases were mutually exclusive and had the same probability for each type of teeth bite (open, normal or deep bite) because all the disease outcomes were equally likely to occur. The percentages were compared with a chi-square test. The p level was set at 0.05 .

The software SPSS 9.0 was employed to evaluate statistical tests.

\section{Results}

A total of 605 children students (mean age $8.5 \pm 2.3$ years; $45 \%$ males; $55 \%$ females) were examined.

Table 1 Distribution of physiological and pathological gait in our population

\begin{tabular}{lllll}
\hline & Normal bite (\%) & Deep-bite (\%) & Open-bite (\%) & Total (\%) \\
\hline Physiological gait (\%) & $86.92 \%(\mathrm{~N}=226)$ & $85.13 \%(\mathrm{~N}=206)$ & $85.30 \%(\mathrm{~N}=87)$ & $100 \%(\mathrm{~N}=519)$ \\
Pathological gait (\%) & $13.08 \%(\mathrm{~N}=34)\left(^{*}\right)$ & $14.87 \%(\mathrm{~N}=36)$ & $14.70 \%(\mathrm{~N}=15)$ & $100 \%(\mathrm{~N}=85)$ \\
Total (\%) & $100 \% \mathrm{~N}=260$ & $100 \%(\mathrm{~N}=242)$ & $100 \%(\mathrm{~N}=102)$ & $\mathrm{N}=604$ \\
\hline
\end{tabular}

* significantly lower than the other two groups. 
Table 2 Distribution of dysmetric legs in our population

\begin{tabular}{lllll}
\hline & Normal bite (\%) & Deep-bite (\%) & Open- bite (\%) & Total (\%) \\
\hline Dysmetric legs (\%) & $6.15 \%(\mathrm{~N}=16)$ & $5.76 \%(\mathrm{~N}=14)$ & $5.94 \%(\mathrm{~N}=6)$ & $100 \%(\mathrm{~N}=36)$ \\
Normal legs (\%) & $93.85 \%(\mathrm{~N}=244)$ & $94.24 \%(\mathrm{~N}=229)$ & $94.06 \%(\mathrm{~N}=95)$ & $100 \%(\mathrm{~N}=568)$ \\
Total $(\%)$ & $100 \% \mathrm{~N}=260$ & $100 \%(\mathrm{~N}=243)$ & $100 \%(\mathrm{~N}=101)$ & $\mathrm{N}=604$ \\
\hline
\end{tabular}

* significantly lower than the other two groups.

Table 1 shows the correlation between gait and dental occlusion (open bite and deep bite).

There was a prevalence of cases with an unphysiological gait in patients with an abnormal overjet (14.7\%) or overbite $(14.87 \%)$, while the percentage of patients with normal occlusion that showed an unphysiological gait was $13.08 \%$.

In our sample, about $85.3 \%-86.9 \%$ of the subjects showed a physiological gait.

Table 2 shows the results for dysmetric legs. In our sample, the percentages of subjects with dysmetric legs were lower than the percentages of subjects with a pathological gait, suggesting that about $5 \%-7 \%$ of the cases with a pathological gait were due to other factors (Table 2). Also, about $93.85 \%-94.2 \%$ of the children had normal legs, without respect to the type of teeth bite (Table 2).

Table 3 shows the data for the ocular dominant test. The majority of subjects had a right dominant eye (386 subjects vs. 221 subjects), which means $63 \%-67 \%$ had a right dominant eye and $33 \%-37 \%$ of the subjects had a left dominant eye. Subjects with an open bite showed a slightly different distribution with a lower difference between the percentages of right or left dominant eyes. In the ocular dominance test (Table 3), patients with an open bite with a right dominant eye vs. left dominant eye were $58.42 \%$ and $41.58 \%$, respectively. Patients with a deep bite that showed a right dominant eye were $66.66 \%$ while $33.33 \%$ had a left dominant eye. Patients with normal occlusion with a right dominant eye were $62.7 \%$ and those with a left dominant eye were $37.3 \%$.

Table 4 shows the results for ocular convergence diseases.

Subjects with an open bite showed a significantly lower percentage $(2.97 \%)$ of CT-eso with respect to subjects with a normal bite or deep bite. Subjects with a deep bite showed a significantly higher percentage of
CT-exo with respect to subjects with a normal or open bite.

Table 5 shows the data for ocular convergence (normal, pathological on the right side or pathological on the left side). Patients with an open bite showed a significantly higher prevalence $(8.65 \%)$ of pathological convergence in the left eye with respect to subjects with a normal or deep bite. Patients with a deep bite showed a significantly lower percentage $(3.70 \%)$ of pathological convergence in the right eye with respect to the other groups.

\section{Discussion}

\section{The dominant eye}

A difference was noted in the observed percentages in the open bite children that showed a left dominant eye $(41.58 \%)$, which was higher than children with a deep bite or normal bite. Deep bite subjects were more likely to show a right dominant eye $(66.6 \%)$ with respect to children with a normal bite or open bite. The data suggested that the tendency of the vertical dimension of the bite (deep bite or open bite) was related to the dominant eye. The relationship between the dominant eye and the mandibular position was reported in terms of change in the transverse plane of the head posture after positioning eye patches over the dominant eye for $1 \mathrm{~h}$ [18].

\section{Ocular convergence disorders (phorias)}

Subjects with an open bite showed a significantly lower percentage (2.97\%) of CT-esophoria with respect to subjects with a normal bite or deep bite. As in other forms of strabismus, esodeviation can be controlled by fusional divergence (esophoria), intermittently controlled by fusional divergence (intermittent esotrophia) or constantly manifest (esotrophia). Subjects with a deep bite showed a significantly higher percentage of CT-exophoria with respect to subjects with a normal or open bite.

Table 3 Distribution of dominant eyes data in our population

\begin{tabular}{|c|c|c|c|c|}
\hline & Normal bite (\%) & Deep-bite (\%) & Open-bite (\%) & Total (\%) \\
\hline Right dominant eye (\%) & $62.70 \%(N=163)$ & $66.66 \%(N=164)$ & $58.42 \%(\mathrm{~N}=59)$ & $100 \%(N=386)$ \\
\hline Left dominant eye (\%) & $37.30 \%(N=97)$ & $33.33 \%(\mathrm{~N}=82)(*)$ & $41.58 \%(\mathrm{~N}=42)(*)$ & $100 \%(N=221)$ \\
\hline Total (\%) & $100 \%(N=260)$ & $100 \%(N=246)$ & $100 \%(N=101)$ & $N=607$ \\
\hline
\end{tabular}

* significantly lower than the other two groups. 
Table 4 Distribution of data about the ocular convergence diseases

\begin{tabular}{|c|c|c|c|c|}
\hline & Normal bite (\%) & Deep-bite (\%) & Open-bite (\%) & Total (\%) \\
\hline CT phoria & $85.38 \%(N=222)$ & $83.53 \%(N=203)$ & $88.12 \%(N=89)$ & $100 \%(N=514)$ \\
\hline CTexophoria & $8.84 \%(N=23)$ & $11.93 \%(\mathrm{~N}=29)\left({ }^{*}\right)$ & $8.91 \%(N=9)$ & $100 \%(N=61)$ \\
\hline CT esophoria & $4.23 \%(N=11)$ & $4.52 \%(\mathrm{~N}=11)$ & $2.97 \%(\mathrm{~N}=3)(*)$ & $100 \%(N=25)$ \\
\hline CT trophia & $1.53 \%(N=4)$ & $0 \%(N=0)$ & $0 \%(N=0)$ & $100 \%(N=4)$ \\
\hline Total (\%) & $100 \%(N=260)$ & $100 \%(N=246)$ & $100 \%(N=101)$ & $N=607$ \\
\hline
\end{tabular}

* significantly lower than the other two groups.

Other studies have shown the relationships of dental occlusion, the oculomotor system and visual stabilization. Evidence for a correlation between eyes and dental occlusion came from the use of mandibular orthopedic repositioning appliances, which simultaneously modify mandibular position and visual focusing tests using prismatic bars. These phenomena gradually disappear after removal of the appliance.

Associations also exist between TMD and oculomotor function. Some authors suggested a much higher prevalence of ocular convergence defects in TMD adults presenting with a limited maximal opening, myofascial pain and pain in the neck and shoulder area [18].

Numerous anatomical connections have been described between trigeminal systems and the nervous structures involved in maintaining posture. The mesencephalic nucleus of the trigeminus, which extends itself from the dorsal portion of the spinal trigeminal nucleus to the caudal part of the superior colliculus, is a sensorial nucleus with unique characteristics.

In the mesencephalic nucleus of the trigeminus, neurons associated with extraocular muscles are present along with the primary afferent neurons associated with the muscles of the MM, tooth pulp and periodontal ligaments. From the mesencephalic nucleus of the trigeminus, the neural pathways connect with the cerebellum, the reticular formation and the medial, inferior, lateral and superior vestibular nuclei.

This cross-sectional study was carried out by observing 605 subjects. Analysis of cross-sectional data usually consists of comparing the differences among the subjects, as well as yielding incidence rates for any single disorder. These data can only describe the incidence proportion of the contemporary presence of occlusal, orthoptic and postural disorders.
In this epidemiological survey, we assessed that:

- About $13 \%$ of the children showed a pathological gait and, among them, patients with vertical anomalies of occlusion (deep bite or open bite) seemed to demonstrate a higher prevalence (with a statistically significant difference of $2 \%$ ) of a pathological gait;

- About $5 \%-6 \%$ of the entire sample demonstrated dysmetric legs, which can explain only $50 \%$ of the cases with a pathological gait;

- The vertical dimension of occlusion revealed a slight relationship with the proper dominant eye, as deep bite patients showed a significantly lower tendency towards a left dominant eye $(\mathrm{p}<0.05)$. Therefore, more were likely to show a right dominant eye. At the same time, deep bite subjects revealed a significantly lower frequency of pathological convergence in their right eye $(\mathrm{p}<0.05)$. Deep bite subjects had the tendency to show more frequent $\mathrm{CT}$ exophoria at a significant level $(\mathrm{p}<0.05)$, while open bite patients showed a significantly lower percentage of CT esophoria.

\section{Conclusions}

In conclusion, postural, orthoptic and occlusal alterations may be clinically associated. Clinical connections or concomitant frequencies can be found among them, often with significant differences. From these data, it is not possible to state that there is a direct causal connection among them, but we may suppose that a causal connection may exist. Therefore, the treatment of such disorders requires the intervention of several specialists and a multidisciplinary approach. It is very important that pediatricians are aware of these possible clinical

Table 5 Distribution of data about the ocular convergence

\begin{tabular}{|c|c|c|c|c|}
\hline & Normal bite (\%) & Deep-bite (\%) & Open-bite (\%) & Total (\%) \\
\hline Normal & $89.18 \%(N=231)$ & $90.94 \%(N=221)$ & $86.54 \%(N=90)$ & $100 \%(N=542)$ \\
\hline Pathological in the right side & $4.24 \%(N=11)$ & $3.70 \%(\mathrm{~N}=9)(*)$ & $4.80 \%(N=5)$ & $100 \%(N=25)$ \\
\hline Pathological in the left side & $6.56 \%(N=17)$ & $5.34 \%(N=13)$ & $8.65 \%(\mathrm{~N}=9)\left({ }^{*}\right)$ & $100 \%(N=39)$ \\
\hline Total (\%) & $100 \%(N=259)$ & $100 \%(N=243)$ & $100 \%(N=104)$ & $N=606$ \\
\hline
\end{tabular}

* significantly lower than the other two groups. 
associations to direct young patients to the appropriate specialists that may treat these various disorders. In addition, it can be concluded that when a child is suffering from one of these disorders (postural, occlusal or orthotic), it may be useful for them to undergo screening for other possibly associated disorders.

\section{Competing interests}

For this study, there was no conflict of interest in: (1) the study design; (2) the collection, analysis and interpretation of data; (3) the writing of the report; and (4) the decision to submit the paper for publication. Armando Silvestrini-Biavati wrote the first draft of the manuscript and no honorarium, grant, or other form of payment was given to anyone to produce the manuscript.

\section{Authors' contributions}

ASB and PSB conceived the study; MM, EDM, ASB and PSB recorded the data; ST analyzed the data; ASB, MM, MS and PSB organized the manuscript and interpreted the results; MS and AP followed the statistical evaluation and reviewed the text; MS and ASB coordinated the work. All authors read and approved the final manuscript.

\section{Acknowledgements}

The authors would like to thank Dr. G. Pittaluga, Dr. L. Berta, Dr. G. Cavallero, Dr. A. Ghidoni (School Medicine-District 1-Genova), who assisted with sample recruitment and administrative school planning, and Dr. F. Aliotta (osteopathist), Dr. E. Artuso (orthoptist), Dr. V. Parodi for their important assistance and work during the clinical examinations.

\section{Author details}

'Department of Orthodontics, University of Genova, Genoa, Italy. ${ }^{2}$ Dental Practitioner, Savona, Italy. ${ }^{3}$ Department of Medical, Oral and Biotechnological Sciences, University G. D'Annunzio Chieti, Chieti, Italy. ${ }^{4}$ Dental Practitioner, Postural Gnathologist, Genoa, Italy. ${ }^{5}$ Department of Pedodontics, Sapienza University of Rome, Rome, Italy.

Received: 28 May 2012 Accepted: 18 January 2013

Published: 23 January 2013

\section{References}

1. Zepa I, Hurmerinta K, Kovero O, Nissinen M, Kononen M, Huggare J: Associations between thoracic kyphosis, head posture, and craniofacial morphology in young adults. Acta Odontol Scand 2000, 58:237-242.

2. Solow B, Sonnesen L: Head posture and malocclusions. Eur J Orthod 1998, 20:685-693

3. Lippold C, Danesh G, Schilgen M, Drerup B, Hackenberg L: Relationship between thoracic, lordotic, and pelvic inclination and craniofacial morphology in adults. Angle Orthod 2006, 76:779-785.

4. Lippold C, Van Den BL, Danesh G, Ehmer U: Interdisciplinary study of orthopedic and orthodontic findings in pre-school infants. J Orofac Orthop 2003, 64:330-340

5. Lippold C, Danesh G, Hoppe G, Drerup B, Hackenberg L: Trunk inclination, pelvic tilt and pelvic rotation in relation to the craniofacial morphology in adults. Angle Orthod 2007, 77:29-35.

6. Andrade AS, Gaviao MB, Gameiro GH, De Rossi M: Characteristics of masticatory muscles in children with unilateral posterior crossbite. Braz Oral Res 2010, 24(2):204-210.

7. Korbmacher H, Eggers-Stroeder G, Koch L, Kahl-Nieke B: Correlations between anomalies of the dentition and pathologies of the locomotor system - a literature review. J Orofac Orthop 2004, 65:190-203.

8. Perillo L, Femminella B, Farronato D, Baccetti T, Contardo L, Perinetti G: Do malocclusion and Helkimo Index $\geq 5$ correlate with body posture? J Oral Rehabil 2011, 38(4):242-252.

9. Perinetti G, Contardo L, Silvestrini-Biavati A, Perdoni L, Castaldo A: Dental malocclusion and body posture in young subjects: A multiple regression study. Clinics 2010, 65(7):689-695.

10. Monaco A, Streni O, Marci MC, Sabetti L, Marzo G, Giannoni M: Relationship between mandibular deviation and ocular convergence. J Clin Pediatr Dent 2004, 28(2):135-138.
11. Sforza C, Tartaglia GM, Solimene U, Morgun V, Kaspranskiy RR, Ferrario VF: Occlusion, sternocleidomastoid muscle activity, and body sway: a pilot study in male astronauts. Cranio 2006, 24(1):43-49.

12. Dogan S, Erturk N: The effect of vision on craniocervical posture and its relation to craniofacial and dentoalveolar morphology. Quintessence Int 1990, 21(5):401-406. No abstract available.

13. Pradham NS, White GE, Mehta N, Forgione A: Mandibular deviations in TMD and non-TMD groups related to eye dominance and head posture. J Clin Pediatr Dent 2001, 25(2):147-155.

14. Herman MJ: Torticollis in infants and children: common and unusual causes. Instr Course Lect 2006, 55:647-653. Review.

15. Strini PJ, Machado NA, Gorreri MC, Ferreira Ade F, Sousa Gda C, Fernandes Neto AJ: Postural evaluation of patients with temporomandibular disorders under use of occlusal splints. J App/ Oral Sci 2009, 17(5):539-543.

16. Silvestrini-Biavati P: Correlazioni fra Occlusione, Postura e Visus. Approccio diagnostico. In Corso di Gnatologia Multimediale, 4B, Vol II. Edited by Cocilovo F, FCF. Caltanisetta: Didattica Multimediale Internazionale; 1999:185-200. ISBN $350-1167-188$.

17. Silvestrini Biavati P: Gli squilibri della Visione. In Compendio di semeiotica occluso posturale. chapter 13. Edited by Toti T. Bologna: Martina; 2007:56-62. ISBN 978 - 88 - 7572 - 056.

18. Lin SY, White GE: Mandibular position and head posture as a function of eye dominance. J Clin Pediatr Dent 1996, 20(2):133-140.

doi:10.1186/1471-2431-13-12

Cite this article as: Silvestrini-Biavati et al:: Clinical association between teeth malocclusions, wrong posture and ocular convergence disorders: an epidemiological investigation on primary school children. $B M C$ Pediatrics 2013 13:12.

\section{Submit your next manuscript to BioMed Central and take full advantage of:}

- Convenient online submission

- Thorough peer review

- No space constraints or color figure charges

- Immediate publication on acceptance

- Inclusion in PubMed, CAS, Scopus and Google Scholar

- Research which is freely available for redistribution 